\title{
Beneficial Effect of the Traditional Chinese Drug Shu-Xue-Tong on Recovery of Spinal Cord Injury in the Rat
}

\author{
Li-Yun Jia, ${ }^{1}$ An-Hui Yao, ${ }^{1}$ Fang Kuang, ${ }^{1}$ Yu-Kai Zhang, ${ }^{1}$ Xue-Feng Shen, ${ }^{2}$ and Gong Ju${ }^{1}$ \\ ${ }^{1}$ Institute of Neurosciences, The Fourth Military Medical University, 17 Changlexi Road, Xi'an 710032, China \\ ${ }^{2}$ Department of Occupational and Environmental Health, Faculty of Military Preventive Medicine, \\ The Fourth Military Medical University, 17 Changlexi Road, Xi'an 710032, China
}

Correspondence should be addressed to Xue-Feng Shen, xfshen@fmmu.edu.cn and Gong Ju, jugong@fmmu.edu.cn

Received 3 October 2009; Revised 20 April 2010; Accepted 30 June 2010

Copyright () 2011 Li-Yun Jia et al. This is an open access article distributed under the Creative Commons Attribution License, which permits unrestricted use, distribution, and reproduction in any medium, provided the original work is properly cited.

\begin{abstract}
Shu-Xue-Tong (SXT) is a traditional Chinese drug widely used to ameliorate stagnation of blood flow, such as brain or myocardial infarction. Whether SXT may have therapeutic value for spinal cord injury (SCI), during which ischemia plays an important role in its pathology, remains to be elucidated. We hypothesized that SXT may promote SCI healing by improving spinal cord blood flow (SCBF), and a study was thus designed to explore this possibility. Twenty-five male Sprague-Dawley rats were used. SCI was induced by compression, and SXT was administrated $24 \mathrm{~h}$ postinjury for 14 successive days. The effects of SXT were assessed by means of laser-Doppler flowmetry, motor functional analysis (open-field walking and footprint analysis), and histological analysis (hematoxylin-eosin and thionin staining and NeuN immunohistochemistry). SXT significantly promoted SCBF of the contused spinal cord and enhanced the recovery of motor function. Histological analysis indicated that the lesion size was reduced, the pathological changes were ameliorated, and more neurons were preserved. Based on these results we conclude that SXT can effectively improve SCI.
\end{abstract}

\section{Introduction}

Mechanical trauma of spinal cord causes focal destruction of neural and vascular tissues that is called primary spinal cord injury (SCI), and this initial insult then instigates a progressive wave of secondary SCI [1]. A plethora of factors may contribute to secondary SCI [2-7], among which ischemia plays an important role [8].

The high metabolic demand of spinal cord is met by its abundant blood flow, and changes in its blood supply may result in devastating outcomes. It has been reported that ischemia results in expanding secondary SCI $[8,9]$, and the degree of ischemia correlates with the severity of injury $[10,11]$. A number of studies have been performed targeting at the hypoperfusion after SCI $[8,10,12-14]$. In our previous study, we found that there were two ischemic zones during the secondary SCI, namely, a zone adjacent to the lesion site, where most of the neurons had disappeared or were degenerating, and a farther zone, in which most of the neurons appeared relatively normal [15]. Apparently, there is little chance to rescue the remained severely degenerating neurons in the zone right next to the injury epicenter. However, it is possible to rescue most of the neurons in the farther zone, which is clinically very important. Therefore, improving spinal cord blood flow (SCBF) may well be a measure to ameliorate secondary SCI.

Shu-Xue-Tong (SXT) is a purified extract of leech and earthworm. The main components of SXT are hirudin and lumbrokinase [16, 17]. Hirudin is a specific thrombin inhibitor $[18,19]$, and lumbrokinase is a strong fibrinolytic enzyme [20]. Both have strong effects on improving blood flow. Thus, SXT has been successfully used to treat patients with cerebral or myocardial infarction $[16,21]$. The effect of SXT on SCI has so far not been studied. The present study was aimed to explore its therapeutic value on the secondary SCI. A compression-induced SCI model was used, and the effects were assessed with laser-Doppler flowmetry, openfield walking test, footprint analysis, and histological and immunohistochemistry staining. We found that SXT could significantly ameliorate secondary SCI. 


\section{Methods}

2.1. Animals. Twenty-five male Sprague-Dawley rats (200$220 \mathrm{~g}$, provided by the Animal Center of the Fourth Military Medical University) were randomly divided into three groups. (1) SCBF assay group: 4 each for saline control and SXT treatment. (2) Motor behavior analysis group: 5 for sham operation, 6 each for saline control and SXT treatment. (3) Morphological analysis group: 6 each for saline control and SXT treatment. All the rats were maintained on a $12 / 12 \mathrm{~h}$ light/dark cycle and were allowed free access to food and water. The procedures were reviewed and approved by the Animal Care Committee of the Fourth Military Medical University. All efforts were made to minimize the number of animals used and their suffering. At the end of the studies, all rats were euthanized with overdose of sodium pentobarbital.

2.2. Surgical Procedure. The rats were anesthetized with $1 \%$ sodium pentobarbital $(50 \mathrm{mg} / \mathrm{kg})$ i.p. A $30-40 \mathrm{~mm}$ dorsal midline incision was made, and bilateral laminectomy was performed at T8 level (corresponding to T9 segment of the spinal cord). According to the transverse diameter of the spinal cord we designed a plastic spinal cord compression plate with a thickness of $0.5 \mathrm{~mm}$ and a breadth of $2.8 \mathrm{~mm}$. The compression plate was attached to a copper rod with a total weight of $20 \mathrm{~g}$. The injury of the spinal cord was produced by applying the compression plate on the dura mater perpendicular to the longitudinal axis of spinal cord. The compression plate was then lowered down ventrally at a rate of $0.5 \mathrm{~mm} / \mathrm{min}$ to the bottom of the vertebral canal and remained there for $5 \mathrm{~min}$. After withdrawal of the compression plate, the skin incision was closed. The operated animals were kept in cages with soft bedding, and the ambient temperature was maintained at $20-23^{\circ} \mathrm{C}$. Manual evacuation of urinary bladder was performed twice daily until an autonomous micturition reflex developed. In the sham group, only bilateral laminectomy was performed.

2.3. Drug Administration. $0.25 \mathrm{~mL} / \mathrm{kg}$ bodyweight per day of SXT (MuDanJiang Youbo Pharmaceutical Co. Ltd., China) was administered intravenously, according to Jin et al. [22]. The time to start the treatment was carefully estimated. The use of SXT, which contains a thrombin inhibitor and a fibrinolytic enzyme, may cause additional bleeding at acute hemorrhagic phase of SCI. The bleeding should have stopped before $24 \mathrm{~h}$. We did pilot experiment to examine if the use of SXT at $24 \mathrm{~h}$ after injury might induce fresh bleeding, which turned out to be not the case. Therefore, the first injection of SXT was started at $24 \mathrm{~h}$ after injury, and thereafter for 14 successive days. For saline controls, the same amount of saline instead of SXT was administered.

2.4. Laser-Doppler Flowmetry. The SCBF was measured using a MoorLab laser-Doppler flowmeter (wavelength, $780 \mathrm{~nm}$ ) and a MP4 probe (Moorlab Instruments, Devon, England) with the afferent and efferent fibers separated by $0.25 \mathrm{~mm}$. The laminectomized animals from the saline and
SXT groups were mounted onto a stereotactic instrument equipped with vertebra fixation device to stabilize the vertebral column. Body temperature was kept at $37.0 \pm$ $0.5^{\circ} \mathrm{C}$ with a heating blanket (Animal Regulator, BME-421A, Institute of Biomedical Engineering, Tianjin, China). The laser-Doppler probe was affixed to a micromanipulator and placed perpendicularly to the spinal cord, barely touching the dorsal surface of the dura mater. The readings of the laser-Doppler signals were recorded into a computer and analyzed with Moorsoft for Windows V 1.31. SCBF was measured before SCI $(0 \mathrm{~h})$ and at $0.5 \mathrm{~h}, 3 \mathrm{~d}$, and $7 \mathrm{~d}$ after SCI in both saline and SXT groups. The measurement taken before SCI was used as the normal control and that taken at $0.5 \mathrm{~h}$ was used to examine SCBF right after the injury. For each later measurement, the dura mater should be exposed, which would aggravate the adhesion between the dura mater and the adjacent tissue. Therefore, SCBF measuring would become impossible after $7 \mathrm{~d}$. Four areas with minimal pial vessels were chosen to measure SCBF, two points on each side at $1 \mathrm{~mm}$ rostral and caudal to the lesion epicenter. The value for each area was taken as the mean of consecutive spikes obtained from $1 \mathrm{~min}$ recording. The final value for each rat was obtained from the average value of the four areas. The mean value of the four regions before injury $(0 \mathrm{~h})$ was taken as the basal value, and the final value for each rat at each later time point was expressed in percentage of the basal value $(100 \%)$.

\subsection{Functional Assessment}

2.5.1. Open-Field Walking Assessment. Motor functional recovery was evaluated at $7 \mathrm{~d}$ and $14 \mathrm{~d}$ using the openfield walking scoring system. The hindlimb movements were scored according to the Tarlov's motor scale: 0 , no spontaneous movement; 1, movement in the hip and/or knee but not ankle; 2, movement of the limb in all three major joints; 3 , active support, uncoordinated gait; 4 , coordination of forelimbs and hindlimbs in gait; 5 , normal gait and base of support, no loss of balance on fast turns, and no toe dragging [23].

2.5.2. Footprint Analysis. The analysis was conducted at $7 \mathrm{~d}$ and $14 \mathrm{~d}$. The plantar surface of both hindlimbs of each rat was colored black and the dorsal surface red with nontoxic inks. After that, the rat was allowed to run towards a dark tunnel on the white paper $\left(21 \times 59.4 \mathrm{~cm}^{2}\right)$ [24]; thus the red and/or black ink was printed onto the paper as one set of footprints. From each set of footprints, the pixels of the black (normal plantar paw placing) and red (abnormal dorsal paw placing) areas were determined using Adobe Photoshop 9.0. The percentage of red pixels defined as red/(black + red $) \times 100 \%$ was computed to quantify toe dragging. Stride length (distance between the centers of ipsilateral adjacent footprints) and stride width (perpendicular distance between the centers of left and right hind limbs) were measured, and the average of five steps in each case was used for statistical comparison. 


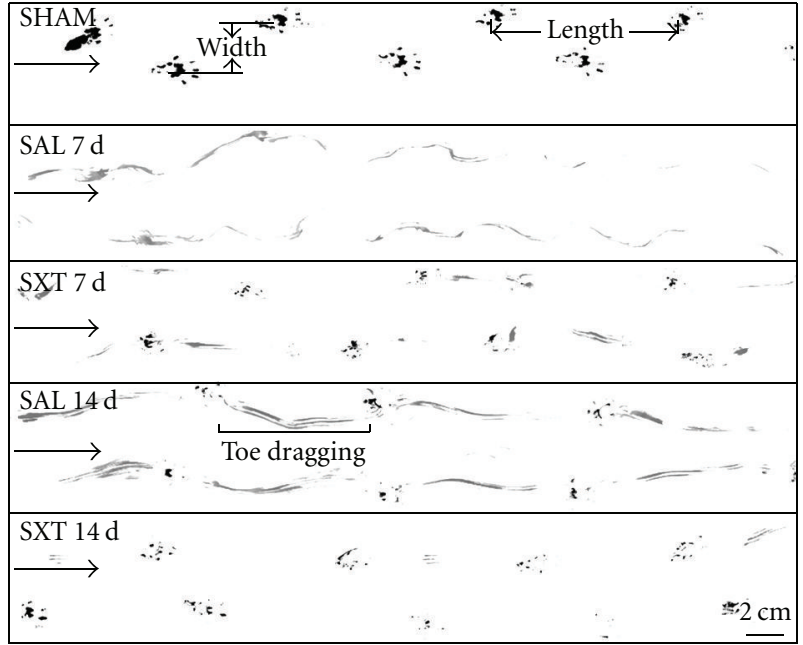

Figure 1: Footprint analysis at 7 and 14 days after injury. Arrows point to the direction of walking. SHAM: sham group; SAL: saline control; SXT: SXT treated group. SHAM: normal walking with normal stride width and length. SAL $7 \mathrm{~d}$ : there is only toe dragging. No plantar stepping appears. SXT $7 \mathrm{~d}$ : plantar prints appear. SAL $14 \mathrm{~d}$ : plantar stepping begins to appear in the saline group. The toe dragging was still serious. SXT $14 \mathrm{~d}$ : the animal resumes almost normal walking. Scale bar $=2 \mathrm{~cm}$.

2.6. Morphological Studies. At $14 \mathrm{~d}$, after footprint analysis, the animals were sacrificed by an overdose of sodium pentobarbital $(100 \mathrm{mg} / \mathrm{kg})$ and perfused intracardially with saline $\left(37^{\circ} \mathrm{C}\right)$ followed by $400 \mathrm{~mL} 4 \%$ cold paraformaldehyde phosphate buffer ( $\mathrm{pH} 7.4$ ). Following perfusion, a $20 \mathrm{~mm}$ spinal cord segment with the injured site at its center was removed and put into $25 \%$ sucrose in phosphate buffer at $4^{\circ} \mathrm{C}$ until the block sank. Serial $20 \mu \mathrm{m}$ frozen sagittal sections were cut on a cryostat and mounted on slides in 10 sets. Hematoxylin and eosin (HE) staining, thionin staining, and NeuN immunostaining were performed, respectively, each on one of the 10 sets. For immunostaining, the sections were rinsed with $0.01 \mathrm{M}$ phosphate buffer saline (PBS) and then blocked with $1 \%$ bovine serum albumin (Sigma) in PBS containing $0.3 \%$ Triton X-100 for $1 \mathrm{~h}$ at room temperature. Sections were then incubated with anti-NeuN antibody ( 1 : 1000; Chemicon, Temecula, CA) for $48 \mathrm{~h}$ at $4^{\circ} \mathrm{C}$. After washing with PBS, the NeuN immunoreactivity was detected by incubating with Alexa Fluor 594 donkey antimouse IgG (1:800; Molecular Probes, Oregon, USA) for $24 \mathrm{~h}$ at $4^{\circ} \mathrm{C}$. Omission of the primary antibody served as the negative control. The sections were observed under an Olympus BX51 microscopy.

\subsection{Quantification and Statistical Analysis}

2.7.1. Area of Injury. HE stained sections were used for analysis of the area of the injury. Five sections were chosen for analysis in each rat, namely, the section with central canal and two adjacent sections on both sides, with $200 \mu \mathrm{m}$ between each section. The microscopic images were obtained with a 40x objective under an Olympus BX-51 microscopy.
The images were opened in Adobe Photoshop 9.0 and stuck together as a montage. The boundary of the injured area was outlined according to the differences between normal tissue and necrotic or apoptotic tissue and captured with the noose tool. The pixel of the outlined area was calculated and then converted to the size of injury.

2.7.2. Count of Neurons. Five sections similar to those mentioned above were chosen for analysis in each rat. The number of NeuN positive cells within $1000 \mu \mathrm{m}$ distance to the injured border was counted with a 200x objective under an Olympus BX-51 microscopy. The mean values for the five sections in each rat were used for statistical analysis.

2.7.3. Statistical Analysis. All data were expressed as mean \pm SD. Values of open-field walking activity were analyzed by the nonparametric Mann-Whitney $U$ test. All the other results were compared with one-way analysis of variance (ANOVA). The statistical program SPSS 12.0 for windows was used for statistic analysis. $P<.05$ was considered significant.

\section{Results}

3.1. Compression-Induced SCI Model. The pial vasculature on the dorsal surface could be clearly identified in the normal spinal cord. After SCI, a transverse hemorrhagic band resulting from compression could be observed on the dorsal surface. HE-stained sagittal sections showed that severe hemorrhage occurred at the lesion epicenter immediately after injury. The compression also caused some bleeding in parts rostral and caudal to the site of compression.

3.2. Spinal Cord Blood Flow. Saline control: laser-Doppler flowmetry assay showed that the SCBF was $58 \pm 6.20 \%$ at $0.5 \mathrm{~h}$ after SCI, $65 \pm 3.81 \%$ at $3 \mathrm{~d}$, and $61 \pm 6.68 \%$ at $7 \mathrm{~d}$. Statistic analysis showed no significant difference among them $(P>.05)$.

SXT Group: the SCBF value increased from $57 \pm 6.43 \%$ at $0.5 \mathrm{~h}$ to $78 \pm 4.16 \%$ at $3 \mathrm{~d}(P<.01)$ and further increased to $84 \pm 2.20 \%$ at $7 \mathrm{~d}(P<.05)$.

3.3. Analysis of Motor Function. Any operation will affect the behavior of the animal. Therefore, for behavioral assessment, a sham group, in which the animals simply receive bilateral laminectomy, was included.

3.3.1. Open-Field Walking Test. It has been proved in the rat that functional recovery could be achieved significantly if as few as $5 \%-10 \%$ white matter was spared [25]. Therefore, in the open-field walking test, we compared the motor functional recovery at the corresponding time points between injured groups, rather than comparing at different time points within the same group. All the rats showed normal hindlimb movements (scored 5) before injury. Immediately after injury, the rats in both injured groups showed complete paraplegia (scored 0 ). However, by $7 \mathrm{~d}$, the SXT group recovered considerable bilateral motor function with a score 


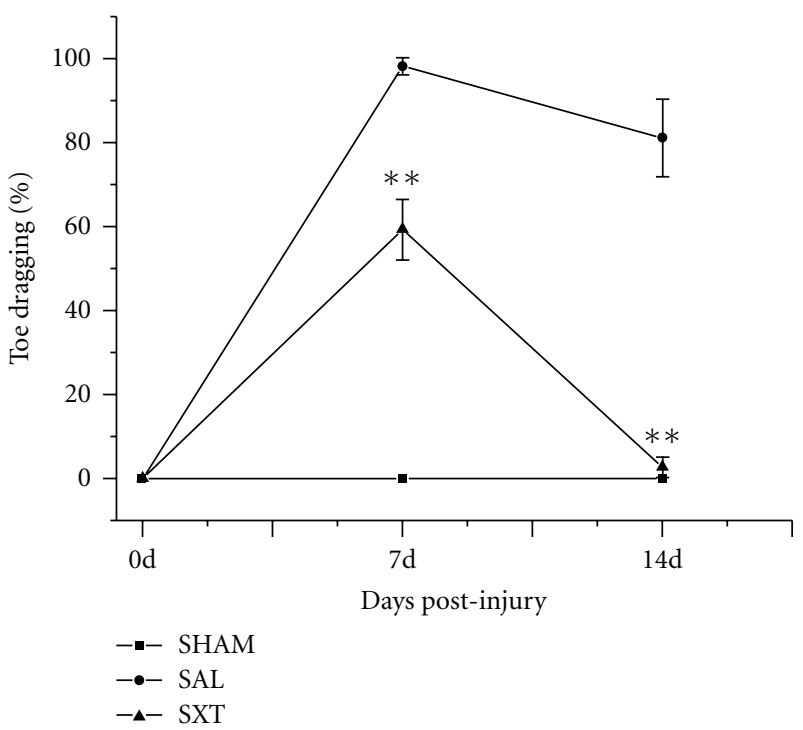

(a)

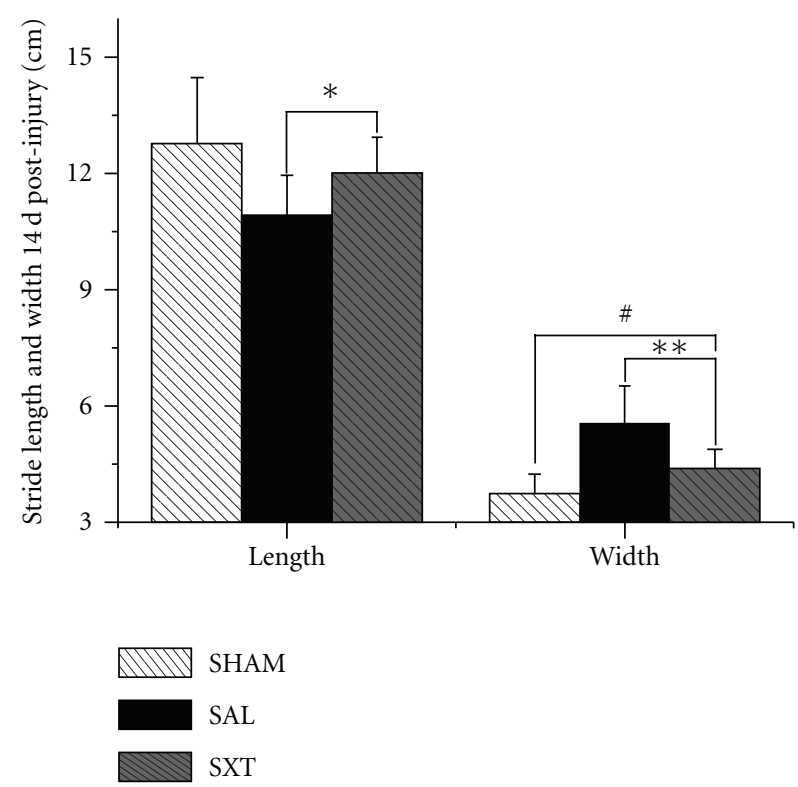

(b)

FIGURE 2: Statistical analysis of footprints. SHAM: Bilaterally laminectomized. 0 d: Preinjury normal control. SAL: saline control, SXT: ShuXue-Tong treated. (a) The SAL group shows no recovery at $7 \mathrm{~d}, 14 \mathrm{~d}$, while the SHAM group has no toe dragging at all. The SXT group resumes significant plantar stepping at $7 \mathrm{~d}$ and is almost normal at $14 \mathrm{~d}$. (b) Both stride width and stride length are significantly improved in the SXT group at $14 \mathrm{~d} .{ }^{* *} P<.01 ;{ }^{*} P<.05 ;{ }^{*} P<.05$.

of $2.83 \pm 0.41$, allowing occasional body weight support, while the saline group only showed extensive movement of hip or knee with no stepping $(1.62 \pm 0.52, P<.01$, compared with SXT group). By $14 \mathrm{~d}$, the SXT group showed a close hindlimb and forelimb coordination $(3.83 \pm 0.41)$. In contrast, the saline group showed a score of $2.67 \pm 0.52$ $(P<.01$, compared with SXT group) with only occasional weight support but no stepping. The sham group showed normal stepping.

3.3.2. Footprint Analysis. The SCI in this study resulted in toe dragging, decrease in stride length, and increase in stride width. Representative running tracings from each treatment were shown in Figure 1. The sham group showed consistent normal plantar stepping during the whole process. At $7 \mathrm{~d}$, severe toe dragging with no planar stepping was observed in the saline group, while the SXT group began to show planar footprints. At $14 \mathrm{~d}$, in the saline group the toe dragging was still serious, and the plantar stepping began to appear, while the SXT group showed improved plantar stepping with a little dorsum prints remaining. In all the time points with plantar stepping, the stride length increased, and the stride width decreased progressively. Also improved were the planter prints, but none was quite complete as compared to the sham group.

Quantitative analysis showed that compared with the saline group, the toe dragging in the SXT group was significantly ameliorated at $7 \mathrm{~d}$ and $14 \mathrm{~d}(59.33 \pm 7.23 \%$ versus $98.22 \pm 1.99 \%$ at $7 \mathrm{~d} ; 2.71 \pm 2.43 \%$ versus $81.14 \pm 9.19 \%$ at $14 \mathrm{~d} ; P<.01$ ) (Figure $2(\mathrm{a})$ ). Because of severe toe dragging with no plantar stepping at $7 \mathrm{~d}$, the stride length and width analysis were only possible in $14 \mathrm{~d}$ studies. Compared with the saline group the stride length in the SXT group was markedly increased $(10.93 \pm 1.02 \mathrm{~cm}$ versus $12.01 \pm 0.93 \mathrm{~cm}$; $P<.05)$ and the stride width significantly reduced $(5.58 \pm$ $0.95 \mathrm{~cm}$ versus $4.39 \pm 0.49 \mathrm{~cm} ; P<.01$ ) (Figure 2(b)). Furthermore, in all the experimental groups the hind limb plantar stepping was laterally rotated.

\subsection{Histological Study}

3.4.1. HE and Thionin Staining. On the $14 \mathrm{~d}$ HE-stained sections, the saline group showed extensive necrosis at the injury site with prominent cavitation (Figure 3(a)). In the SXT-treated group the lesion size was significantly smaller, and the cavitation was milder (Figure 3(b)). The area of the lesion was $4.01 \pm 0.81 \mathrm{~mm}^{2}$ in the saline group and $2.67 \pm 0.36 \mathrm{~mm}^{2}$ in the SXT group $(P<.01)$. The histopathological changes displayed in the thionin-stained sections demonstrated that the SXT group showed more neurons and less inflammatory cells in the area adjacent to the lesion site compared with the saline group (Figures 3(d)$3(\mathrm{~g}))$.

3.4.2. NeuN Immunohistochemistry. The number of NeuNimmunoreactive neurons in an area $1000 \mu \mathrm{m}$ away from the edge of the lesion was counted. Compared to the saline group $(37.2 \pm 9.60)$, a significantly larger number of neurons were present in the SXT group $(56 \pm 10.79, P<.05)$ (Figures $4(\mathrm{a})-$ $4(\mathrm{c})$ ).

The beneficial effect of SXT in ameliorating the secondary injury is summarized in Figure 5. 


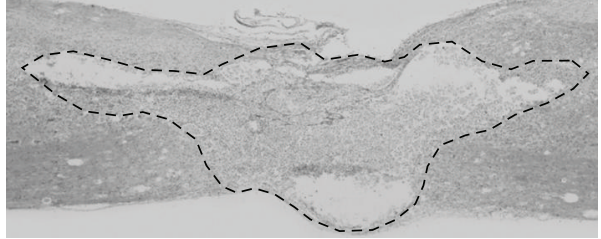

(a)

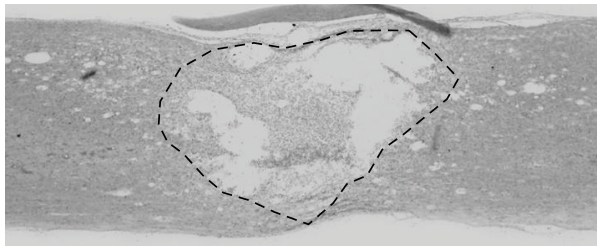

(b)

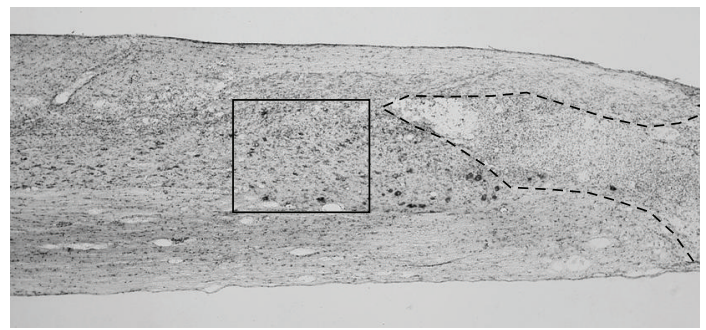

(d)

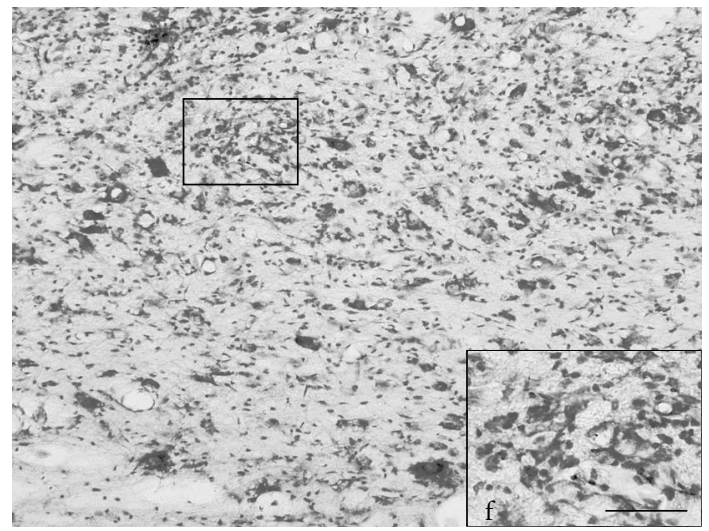

(f)

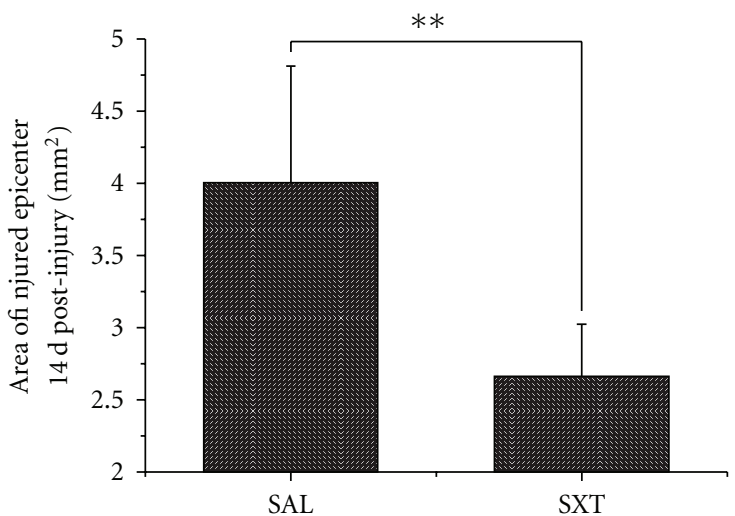

(c)

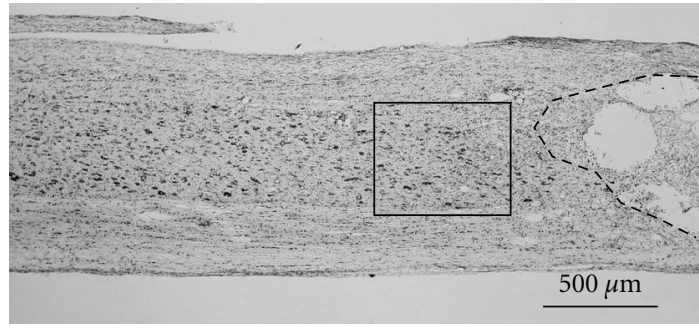

(e)

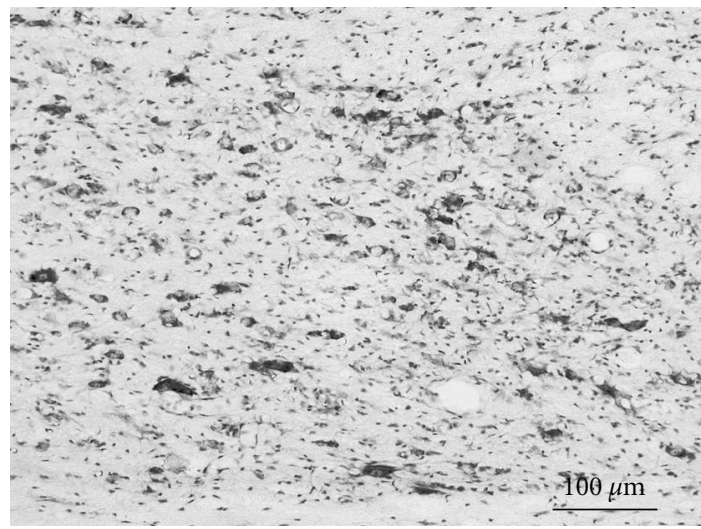

(g)

Figure 3: HE and thionin staining at $14 \mathrm{~d}$. (a) and (b) HE staining. (a) Saline control, (b) SXT treated. The boundary of the lesion site is outlined. The lesion area is significantly smaller in (b). (c) The lesion area is prominently reduced in the SXT group $(P<.01)$. (d) $-(\mathrm{g})$ Thionin staining. (d) and (f) Saline control. (e) and (g) SXT treated. The boxes in (d), (e) are the areas adjacent to the lesion sites, showing that there is larger number of neurons in the SXT treated animal. (f) and (g) are successive blown-ups of the boxes in (d) and (e), displaying the abundance of microglia cells in the saline control. ( $\mathrm{f}$ in (f) is the high magnification of the box at the upper left part of the figure). Scale bar in (a), (b) and (d) $-(\mathrm{e})=500 \mu \mathrm{m}$; in (f) and (g) $=100 \mu \mathrm{m}$; in (f) $=25 \mu \mathrm{m}$.

\section{Discussion}

The main causes of SCI are traffic accidents, fall from a hight, and weight crushing. In many cases the fracture or dislocation of vertebral column results in compression or hyperextension of the spinal cord [26], resulting in paralysis of limbs. The spinal cord is rarely totally transected even after severe contusive injury associated with complete paralysis $[5,27]$. It has been proved that sparing as few as $5 \%-$ $10 \%$ of the fibers in the spinal cord is sufficient to facilitate basic locomotion recovery following SCI injury in rats [25]. That the majority of SCI are incomplete and that a small percentage of spared fibers are sufficient for resumption of basic locomotion encourage researches for the treatment of SCI [28-30].

A major clinical concern is to minimize secondary degeneration following SCI. During the process of secondary SCI, there exists a penumbral ischemic zone adjacent to the lesion epicenter, and this region exhibits progressive cell death over time [8]. We found two zones next to the 


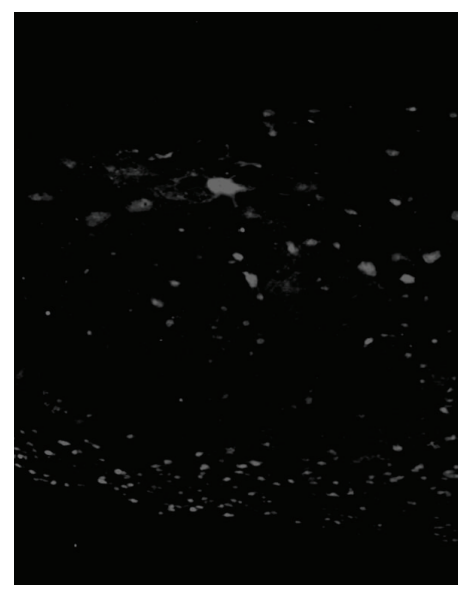

(a)

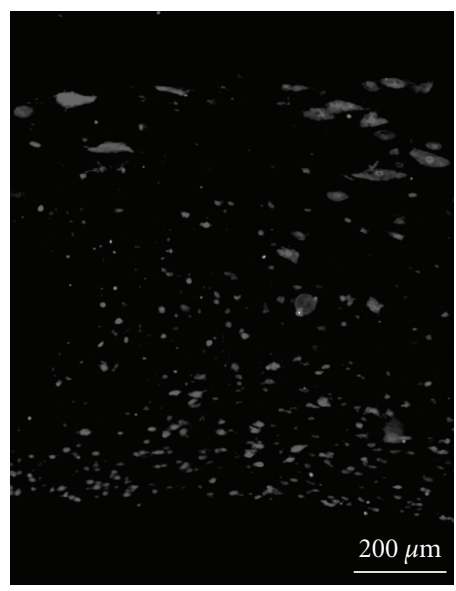

(b)

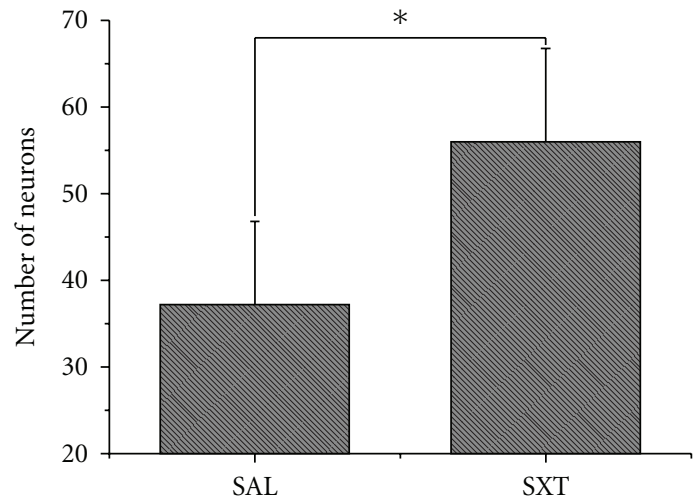

(c)

Figure 4: NeuN immunohistochemistry at $14 \mathrm{~d}$. (a) Saline control. (b) SXT treated. The photos of two areas of similar size were taken $1000 \mu \mathrm{m}$ away from the lesion. Note that there is larger number of neurons in the SXT group. Scale bar $=200 \mu \mathrm{m}$. (c) As compared to the SAL group, the number of neurons is significantly higher in the SXT group $(P<.05)$.

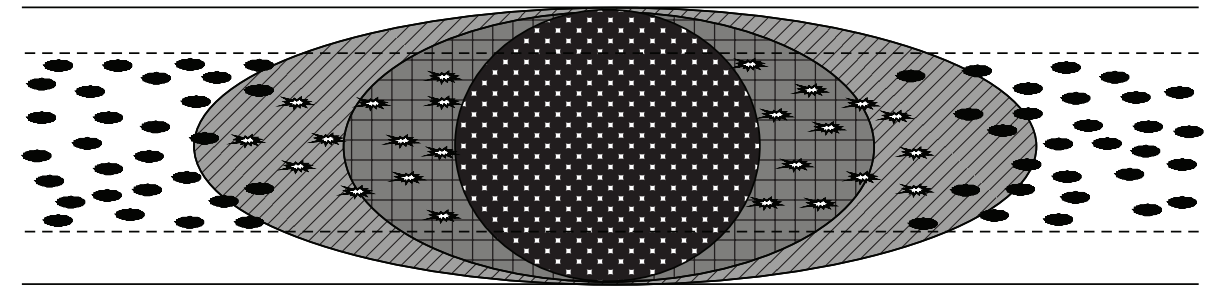

(a)

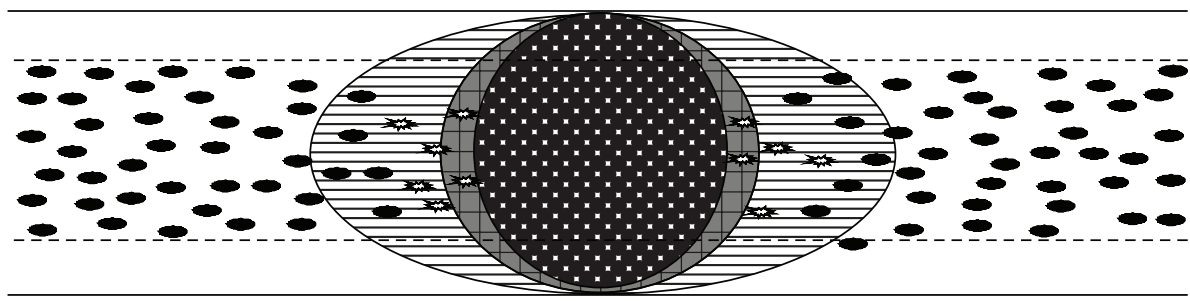

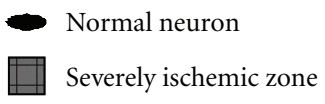

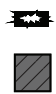

Degenerating neuron

Moderately ischemic zone
Necrotic core

Ameliorated ischemic

(b)

FIGURE 5: Diagrammatic summary of the beneficial effect of SXT in ameliorating the secondary injury.

injury epicenter with different degrees of ischemia, which brings us hope that neurons in the farther zone can be rescued by enhancing the SCBF [15]. In this study SXT was used to promote blood flow in the spinal cord. It has been widely used in China to treat patients suffering from various infarctions, such as cerebral or myocardial infarction $[16,21]$. The main effect of SXT is to improve blood flow through its two components, namely, hirudin and lumbrokinase [17]. Our results demonstrated that SXT promoted SCBF in crushed spinal cord, facilitated motor functional recovery, decreased the lesion size, and rescued neurons in the ischemic area near the lesion site. One of the beneficial effects of acupuncture in treating spinal cord injuries has been reported to be the increase in SCBF [31, 32]. It lends support to our present study. Moreover, it has been reported that one of the major components of SXT, hirudin, being a specific thrombin inhibitor, is able to reduce thrombin-induced brain edema $[18,19]$. Thus, it may well be possible that the beneficial effect of SXT on functional recovery in SCI is in part credited to the reduction of spinal cord edema.

In conclusion, the present study demonstrated that SXT can significantly promote SCBF, minimize secondary injury, protect neurons in the ischemic area, and facilitate motor 
recovery. These findings laid a basis for future clinical application of SXT for the treatment of SCI.

\section{Acknowledgments}

The authors thank Yi-hui Liu and Xi-ying Jiao for their technical helps. This work was supported by the Natural Science Foundation of China (2003CB515301), the Military 11th Five-year Special-Purpose Program for PLA (082028), the Chinese PLA National Scientific Technological Project (06G089), the Major Project of Military Medicine (08XJZ002), and the Military 11th Five-year Development of Medicine (2006K15-G1).

\section{References}

[1] J. W. McDonald and C. Sadowsky, "Spinal-cord injury," Lancet, vol. 359, no. 9304, pp. 417-425, 2002.

[2] J. M. Schwab, K. Brechtel, C.-A. Mueller et al., "Experimental strategies to promote spinal cord regeneration-an integrative perspective," Progress in Neurobiology, vol. 78, no. 2, pp. 91116,2006

[3] C. Profyris, S. S. Cheema, D. Zang, M. F. Azari, K. Boyle, and S. Petratos, "Degenerative and regenerative mechanisms governing spinal cord injury," Neurobiology of Disease, vol. 15, no. 3, pp. 415-436, 2004.

[4] G. D. Carlson and C. Gorden, "Current developments in spinal cord injury research," Spine Journal, vol. 2, no. 2, pp. 116-128, 2002.

[5] R. Nashmi and M. G. Fehlings, "Mechanisms of axonal dysfunction after spinal cord injury: with an emphasis on the role of voltage-gated potassium channels," Brain Research Reviews, vol. 38, no. 1-2, pp. 165-191, 2001.

[6] A. Josephson, D. Greitz, T. Klason, L. Olson, and C. Spenger, "A spinal thecal sac constriction model supports the theory that induced pressure gradients in the cord cause edema and cyst formation," Neurosurgery, vol. 48, no. 3, pp. 636-646, 2001.

[7] B. K. Kwon, W. Tetzlaff, J. N. Grauer, J. Beiner, and A. R. Vaccaro, "Pathophysiology and pharmacologic treatment of acute spinal cord injury," Spine Journal, vol. 4, no. 4, pp. 451464, 2004.

[8] A. E. Mautes, M. R. Weinzierl, F. Donovan, and L. J. Noble, "Vascular events after spinal cord injury: contribution to secondary pathogenesis," Physical Therapy, vol. 80, no. 7, pp. 673-687, 2000.

[9] J. D. Balentine, "Pathology of experimental spinal cord trauma. I. The necrotic lesion as a function of vascular injury," Laboratory Investigation, vol. 39, no. 3, pp. 236-253, 1978.

[10] M. G. Fehlings, C. H. Tator, and R. D. Linden, "The relationships among the severity of spinal cord injury, motor and somatosensory evoked potentials and spinal cord blood flow," Electroencephalography and Clinical Neurophysiology, vol. 74, no. 4, pp. 241-259, 1989.

[11] Y. Hamamoto, T. Ogata, T. Morino, M. Hino, and H. Yamamoto, "Real-time direct measurement of spinal cord blood flow at the site of compression: relationship between blood flow recovery and motor deficiency in spinal cord injury," Spine, vol. 32, no. 18, pp. 1955-1962, 2007.

[12] A. Holtz, B. Nystrom, and B. Gerdin, "Relation between spinal cord blood flow and functional recovery after blocking weight-induced spinal cord injury in rats," Neurosurgery, vol. 26, no. 6, pp. 952-957, 1990.

[13] D. L. Anthes, E. Theriault, and C. H. Tator, "Ultrastructural evidence for arteriolar vasospasm after spinal cord trauma," Neurosurgery, vol. 39, no. 4, pp. 804-814, 1996.

[14] G. Guízar-Sahagún, A. Ibarra, A. Espitia, A. Martínez, I. Madrazo, and R. E. Franco-Bourland, "Glutathione monoethyl ester improves functional recovery, enhances neuron survival, and stabilizes spinal cord blood flow after spinal cord injury in rats," Neuroscience, vol. 130, no. 3, pp. 639-649, 2005.

[15] X.-F. Shen, Y. Zhao, Y.-K. Zhang, L.-Y. Jia, and G. Ju, "A modified ferric tannate method for visualizing a blood vessel and its usage in the study of spinal cord injury," Spinal Cord, vol. 47, pp. 852-856, 2009.

[16] A. H. Li, K. Z. Gong, J. F. Yan, X. Sun, Y. Feng, and Z. Zhang, "Effect of shuxuetong in preventing restenosis after intracoronary stenting," Chinese Journal of Integrated Traditional and Western Medicine, vol. 24, no. 10, pp. 879-881, 2004 (Chinese).

[17] X. Zhang, B. Xiao, and C.-L. Hu, "Study in the mechanism of Shuxuetong injection on antithrombosis and thrombolysis," Zhongguo Zhongyao Zazhi, vol. 30, no. 24, pp. 1950-1952, 2005 (Chinese).

[18] G. P. Colon, K. R. Lee, R. F. Keep, T. L. Chenevert, A. L. Betz, and J. T. Hoff, "Thrombin-soaked gelatin sponge and brain edema in rats," Journal of Neurosurgery, vol. 85, no. 2, pp. 335339, 1996.

[19] K. Kawakita, N. Kawai, Y. Kuroda, S. Yasashita, and S. Nagao, "Expression of matrix metalloproteinse-9 in thrombininduced brain edema formation in rats," Journal of Stroke and Cerebrovascular Diseases, vol. 15, no. 3, pp. 88-95, 2006.

[20] H. Mihara, H. Sumi, T. Yoneta et al., "A novel fibrinolytic enzyme extracted from the earthworm, Lumbricus rubellus," Japanese Journal of Physiology, vol. 41, no. 3, pp. 461-472, 1991.

[21] K. J. Li and L. J. Miao, "Evaluation of Shuxuetong injection for acute ischaemic stroke," Clinical Medicine of China, vol. 23, pp. 7-10, 2007.

[22] X. Jin, G. Shen, F. Gao et al., "Traditional Chinese drug ShuXueTong facilitates angiogenesis during wound healing following traumatic brain injury," Journal of Ethnopharmacology, vol. 117, no. 3, pp. 473-477, 2008.

[23] D. L. Behrmann, J. C. Bresnahan, M. S. Beattie, and B. R. Shah, "Spinal cord injury produced by consistent mechanical displacement of the cord in rats: behavioral and histologic analysis," Journal of Neurotrauma, vol. 9, no. 3, pp. 197-217, 1992.

[24] S. Saadoun, B. A. Bell, A. S. Verkman, and M. C. Papadopoulos, "Greatly improved neurological outcome after spinal cord compression injury in AQP4-deficient mice," Brain, vol. 131, no. 4, pp. 1087-1098, 2008.

[25] D. M. Basso, M. S. Beattie, and J. C. Bresnahan, "Graded histological and locomotor outcomes after spinal cord contusion using the NYU weight-drop device versus transection," Experimental Neurology, vol. 139, no. 2, pp. 244-256, 1996.

[26] C. Profyris, S. S. Cheema, D. Zang, M. F. Azari, K. Boyle, and S. Petratos, "Degenerative and regenerative mechanisms governing spinal cord injury," Neurobiology of Disease, vol. 15, no. 3, pp. 415-436, 2004.

[27] B. A. Kakulas, "Pathology of spinal injuries," Central Nervous System Trauma, vol. 1, no. 2, pp. 117-129, 1984. 
[28] B. K. Kwon, C. G. Fisher, M. F. Dvorak, and W. Tetzlaff, "Strategies to promote neural repair and regeneration after spinal cord injury," Spine, vol. 30, no. 17, pp. S3-S13, 2005.

[29] L. McKerracher, "Spinal cord repair: strategies to promote axon regeneration," Neurobiology of Disease, vol. 8, no. 1, pp. 11-18, 2001.

[30] H. Zhu, Y.-P. Feng, Y. Wise et al., "Early neurosurgical intervention of spinal cord contusion: an analysis of 30 cases," Chinese Medical Journal, vol. 121, no. 24, pp. 2473-2478, 2008.

[31] S. Uchida and H. Hotta, "Acupuncture affects regional blood flow in various organs," Evidence-Based Complementary and Alternative Medicine, vol. 5, no. 2, pp. 145-151, 2008.

[32] P. T. Dorsher and P. M. McIntosh, "Acupuncture's effects in treating the sequelae of acute and chronic spinal cord injuries: a review of allopathic and traditional chinese medicine literature," Evidence-Based Complementary and Alternative Medicine, vol. 25, pp. 1-8, 2009. 


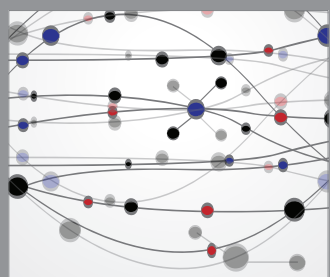

The Scientific World Journal
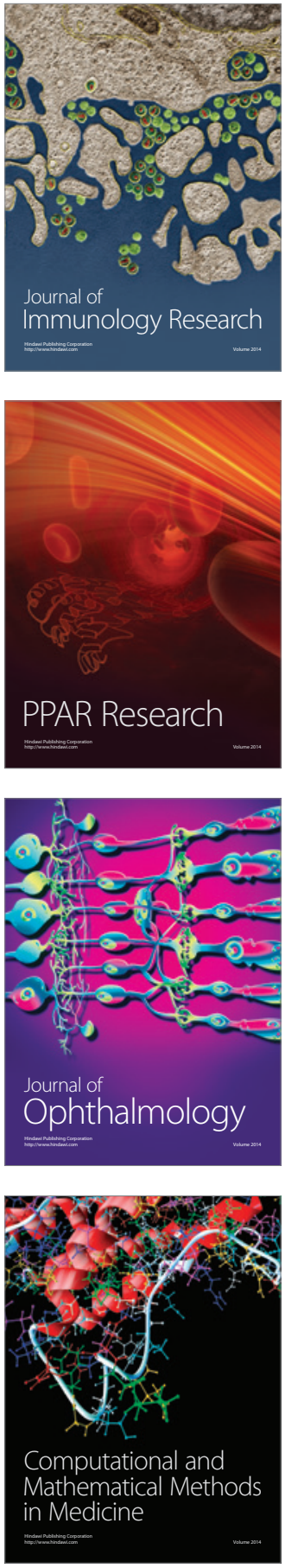

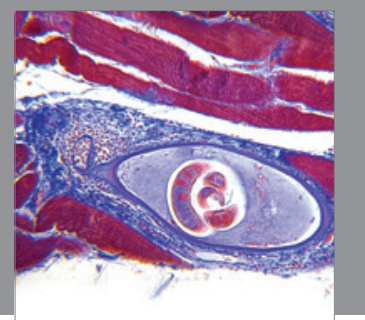

Gastroenterology

Research and Practice
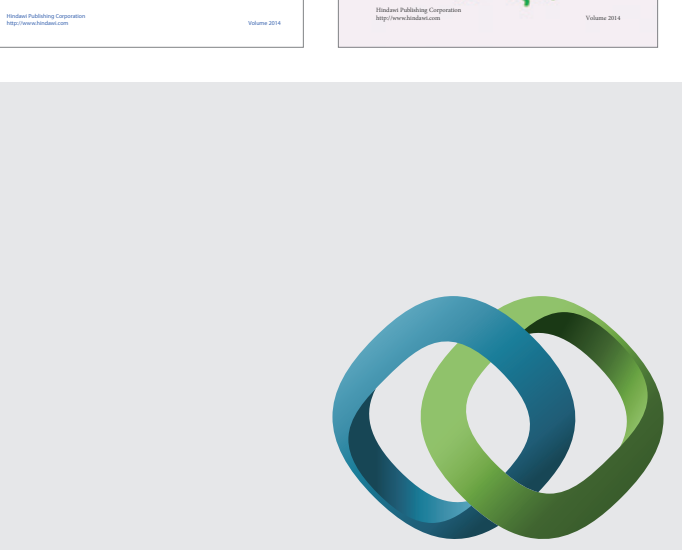

\section{Hindawi}

Submit your manuscripts at

http://www.hindawi.com
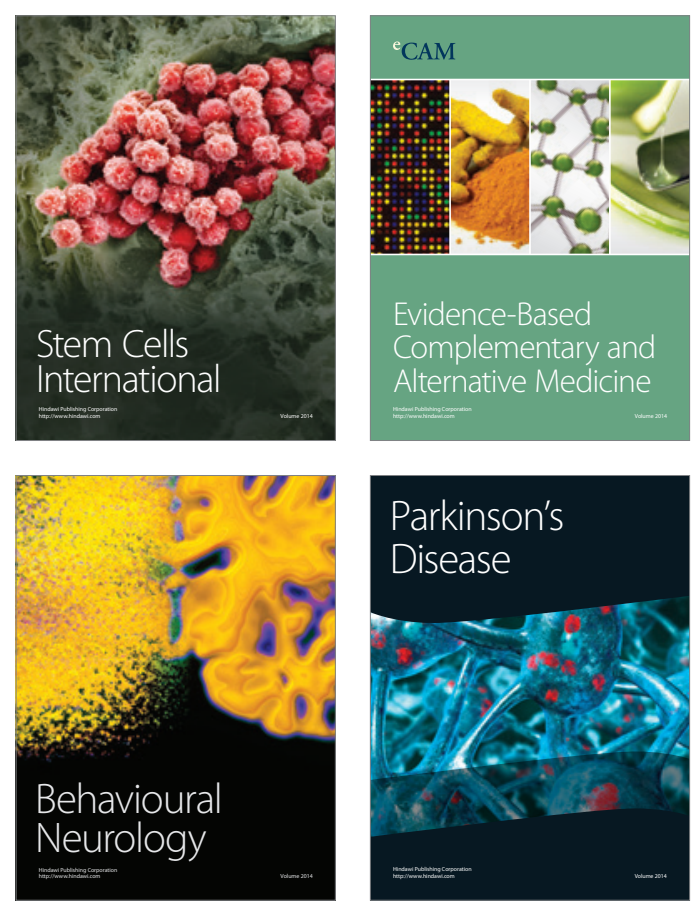

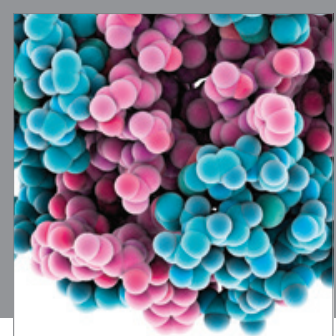

Journal of
Diabetes Research

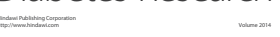

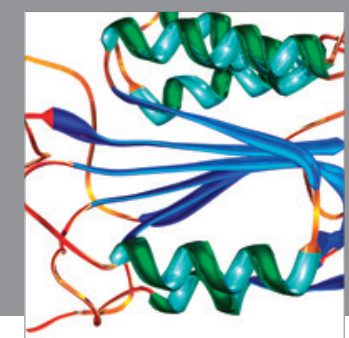

Disease Markers
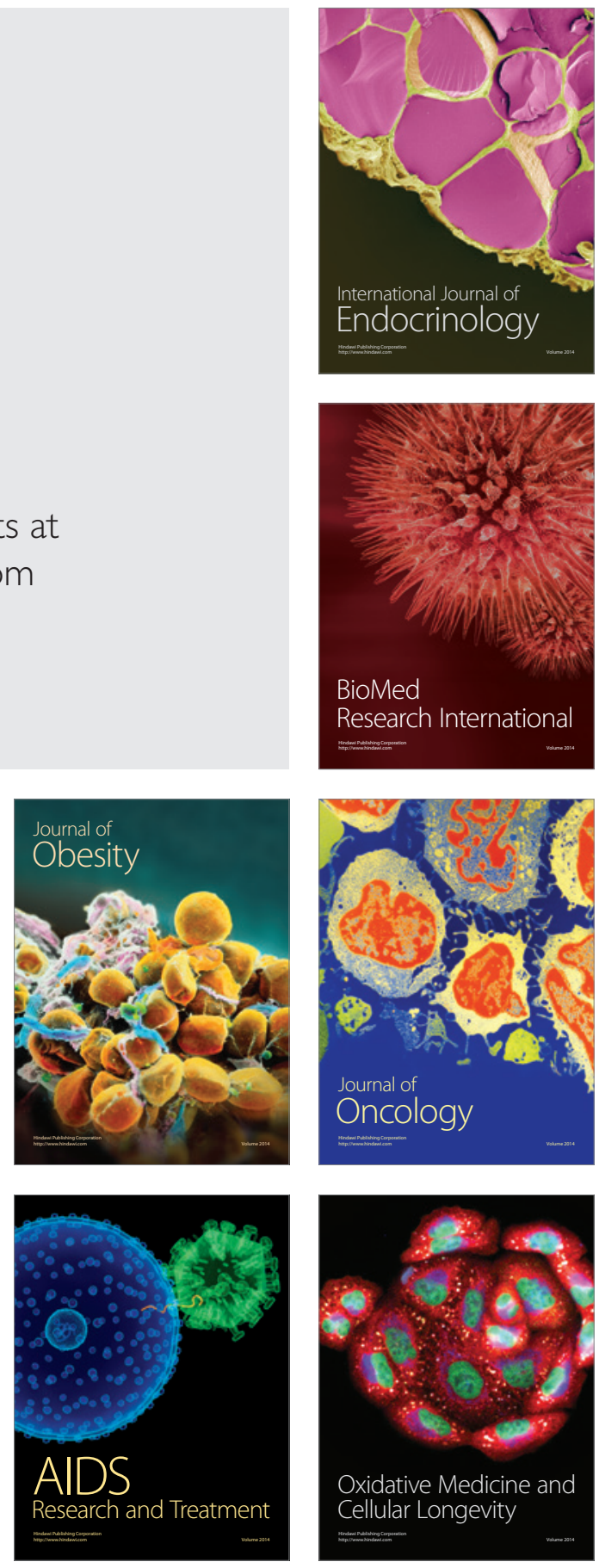